\title{
Optimization of DP-M-QAM Transmitter using Cooperative Coevolutionary Genetic Algorithm
}

Medeiros Diniz, Júlio César; Da Ros, Francesco; Porto da Silva, Edson; Jones, Rasmus Thomas; Zibar, Darko

Published in:

Journal of Lightwave Technology

Link to article, DOI:

10.1109/JLT.2018.2815347

Publication date:

2018

Document Version

Peer reviewed version

Link back to DTU Orbit

Citation (APA):

Medeiros Diniz, J. C., Da Ros, F., Porto da Silva, E., Jones, R. T., \& Zibar, D. (2018). Optimization of DP-MQAM Transmitter using Cooperative Coevolutionary Genetic Algorithm. Journal of Lightwave Technology, 36(12), 2450 - 2462. https://doi.org/10.1109/JLT.2018.2815347

\section{General rights}

Copyright and moral rights for the publications made accessible in the public portal are retained by the authors and/or other copyright owners and it is a condition of accessing publications that users recognise and abide by the legal requirements associated with these rights.

- Users may download and print one copy of any publication from the public portal for the purpose of private study or research.

- You may not further distribute the material or use it for any profit-making activity or commercial gain

- You may freely distribute the URL identifying the publication in the public portal 


\title{
Optimization of DP-M-QAM Transmitter using Cooperative Coevolutionary Genetic Algorithm
}

\author{
Júlio César Medeiros Diniz, Student Member, OSA, Francesco Da Ros, Member, OSA, IEEE, \\ Edson Porto da Silva, Member, OSA, IEEE, Rasmus Thomas Jones, and Darko Zibar, Member, IEEE
}

\begin{abstract}
We present a method for joint optimization of transmitter in-phase, quadrature and inter-polarization time skew, amplitude mismatch, and bias voltages. The method is based on a cooperative coevolutionary genetic algorithm with fitness functions extracted from a directly detected reference QAM signal generated at the transmitter. As a calibration method, it is able to find the values that will statically generate the best possible constellation. To the extent of the simulation investigations conducted, the algorithm is capable to calibrate time skews, bias voltages, IQ phase imbalances, and relative amplitude imbalances with standard deviation of residual error as low as $0.24 \mathrm{ps}, 0.019 \mathrm{~V}, \mathbf{0 . 5 6}^{\circ}$, and 0.003 , respectively, for a dual polarization IQ modulator with $V_{\pi}=4 \mathrm{~V}$ and a 16QAM reference signal operating at $16 \mathrm{GBd}$. An experimental demonstration is also reported.
\end{abstract}

Index Terms-Fiber optics and optical communications, coherent communications.

\section{INTRODUCTION}

$\mathbf{H}$ IGH capacity optical transmission has been widely investigated for long-haul links [1]. High-order modulation formats at high symbol rates combined with coherent detection are of particular interest due to their ability to meet the growing demands for higher bit rates while still reducing the cost per bit. The constant development in electronics enabled state-of-the-art transceivers to evolve from legacy intensitymodulation and direct-detection schemes to a combination of multilevel modulation formats and coherent detection employing digital signal processing (DSP) [2]-[4].

Due to the physical complexity of a typical coherent optical transceiver, it is common that imperfections affect the signal at both the transmitter and the receiver. On the transmitter side, imperfections may arise due to time skews, phase and gain imbalances, arbitrary DC levels and intrinsic modulator nonlinearities. With the requirements becoming more stringent as we move towards even higher modulation formats (> 64QAM) and symbol rates (> $32 \mathrm{GBd})$.

DSP-based coherent optical receivers generally employ an adaptive dynamic equalizer based on a complex-valued

Manuscript received November 29, 2017; revised February 6, 2018; accepted March 7, 2018. Date of publication MM DD, 2018; date of current version MM DD, 2018. This work was funded by Villum Foundation, Søborg, Denmark.

The authors are with DTU Fotonik, Department of Photonics Engineering, Technical University of Denmark, Kongens Lyngby, DK-2800, Denmark (e-mail: \{jcmdi;fdro;edpod;rajo;dazi $\} @$ fotonik.dtu.dk)

Color versions of one or more of the figures in this letter are available online at http://ieeexplore.ieee.org.

Digital Object Identifier 10.1109/JLT.2018.XXXXXXX multiple-input-multiple-output (MIMO) architecture for polarization demultiplexing [3]. It is well known that these equalizers are able to compensate for time skew between polarizations, but can not compensate for time skews between in-phase and quadrature (IQ) components or for IQ imbalances [5]. Although some adaptive higher-order MIMO equalizers robust to IQ time skews and IQ imbalances were proposed [5]-[8], such algorithms increase the already stressed receiver complexity, leading to increased power consumption. Another proposal uses a blind source adaptive separation method to avoid the increased complexity of these high-order MIMO [9]. However, none of these methods take into consideration the increased jitter inserted in the timing synchronization due to IQ time skews and IQ imbalances present at the received signal [10]. Additionally, it is very difficult to separate the imperfections coming from the transmitter from the ones coming from the receiver, reducing the applicability of these methods as just calibration methods.

As time skews and imbalances are static or very slow drifting impairments, the use of adaptive equalization is unnecessary. Thus, it is preferable to estimate and compensate for the IQ phase and amplitude imbalances and the time skews from the transmitter statically, rerunning the calibration process periodically to cope with aging of equipment. Recently, some algorithms to solve these problems were presented. For the time skew estimation, a method based on re-configurable interference was proposed [11]. However, this method needs a special apparatus based on integrated photonics. A method based on generation of arbitrary sine waves for self-calibration [12] and a method based on clock tone amplitude (CTA) extraction of a direct-detected signal, with the searching procedure done by a genetic algorithm, were also proposed [13]. These methods can suffer if the transmitter bias voltages are not well set.

The main goal of this paper is to propose a method to automatically estimate and compensate for the front-end imperfections present in a dual polarization (DP) optical transmitter for high-order QAM modulation formats ( $M$-QAM). Then, we propose an optimization method to mitigate possible amplitude mismatches and time skews between signal components, as well as to find the optimum values for the bias voltages of a DP- $M$-QAM transmitter by employing information derived from directly detected signals making this method simple and cost effective. This method is based on a cooperative coevolutionary genetic algorithm that converges to the best solution through fitness functions extracted from the the directly detected reference QAM signal. We analyze the proposed 
method through extensive simulations, and demonstrate it through experiments.

This paper is divided as follows. In Section II, we present and review a theoretical framework for the optical high-order QAM modulation format generation, the impairments that usually affect this type of transmitter and the information that can be extracted by direct detection of the generated signal. In Section III, we present the proposed coevolutionary genetic algorithm based method for transmitter impairments calibration. In Section IV, we analyze through simulations the proposed method and demonstrate the estimation capabilities of this method through experiments. Finally, the paper is concluded in Section V.

\section{THEORETICAL FRAMEWORK}

In this section, we show common impairments that may occur in the generation process and discuss on how to extract the information about these impairments through direct detection, and subsequent compensation methods. More specifically, we discuss the generation of an optical signal employing highorder QAM modulation formats in subsection A, and the time skew is presented in subsection B. A discussion about the effect of improper bias voltages in QAM signals is presented in subsection C. Finally, in section D, a definition about the amplitude mismatch and an estimation method are presented.

\section{A. Generation of high-order QAM optical signals}

In high symbol rate optical communication systems, highorder modulation formats are typically generated by employing an in-phase and quadrature (IQ) modulator. This modulator is typically composed of two parallel Mach-Zehnder modulators (MZM) operating in the push-pull mode and embedded inside another MZM [14], as shown in Fig. 1.

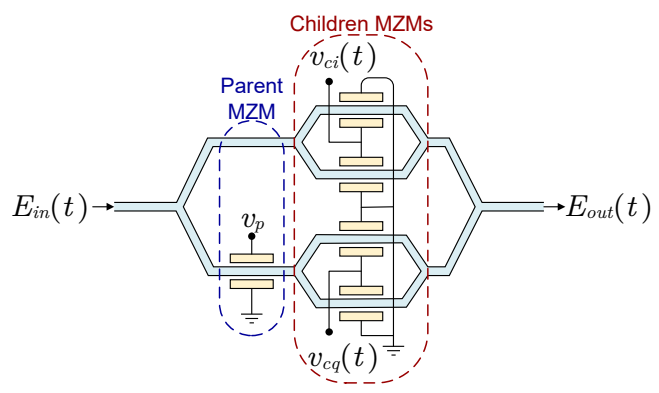

Figure 1. In-phase-quadrature modulator.

The internal MZMs are known as the children modulators and they are used to generate the in-phase and quadrature components of the signal. The external MZM is used to control the phase between the two components of the signal, orthogonalizing them. This external MZM is also known as the parent modulator. Omitting power losses, the relation between the optical carrier electrical field, $E_{i n}(t)$, and the modulated output signal, $E_{\text {out }}(t)$, in an IQ modulator is given by

$$
\begin{aligned}
E_{\text {out }}(t)=\frac{E_{\text {in }}(t)}{2}[ & \cos \left(\frac{\pi v_{c i}(t)}{2 V_{\pi}}\right)+ \\
& \left.\quad \exp \left(j \frac{\pi}{V_{\pi}} v_{p}\right) \cos \left(\frac{\pi v_{c q}(t)}{2 V_{\pi}}\right)\right],
\end{aligned}
$$

where $V_{\pi}$ is the required voltage to delay the phase of an optical signal in a branch by $\pi$ rad. The parent bias, $v_{p}$, is the voltage that controls the phase between in-phase and quadrature components of the signal, being necessary to guarantee a $\pi / 2$ phase shift orthogonality. The electrical modulating driving signals, $v_{c i}(t)$ and $v_{c q}(t)$, are given by

$$
\begin{gathered}
v_{c i}(t)=\dot{v}_{c i}+\ddot{v}_{c i}(t), \\
v_{c q}(t)=\dot{v}_{c q}+\ddot{v}_{c q}(t),
\end{gathered}
$$

where $\ddot{v}_{c i}(t)$ and $\ddot{v}_{c q}(t)$ are the electrical waveforms carrying information, and $\dot{v}_{c i}$ and $\dot{v}_{c q}$ are the children bias voltages (also known as operation points).

Ideally, in order to generate a standard single polarization 4QAM signal, the parent bias should be set as $v_{p}=V_{\pi} / 2$, with the driving signals swinging with peak-to-peak voltages of $2 V_{\pi}$ around the children bias voltages of $\dot{v}_{c i}=\dot{v}_{c q}=-V_{\pi}$. Thus, the output signal has the maximum power efficiency and maximum extinction ratio. The electrical field transfer function of the children MZM for this configuration is shown in Fig. 2(a1), and the resultant constellation is shown in Fig. 2(a2).
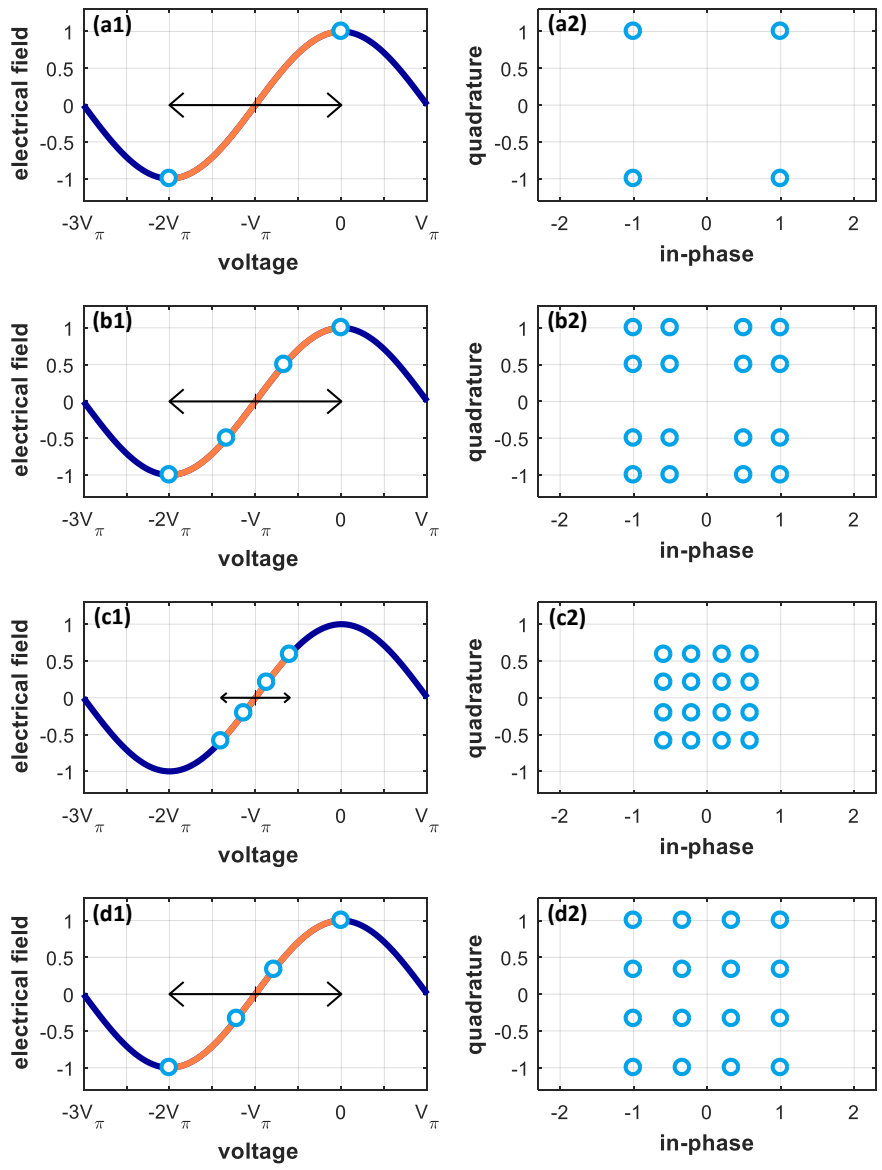

Figure 2. Exemplary single-polarization QAM generation. (1) Electrical field transfer functions. (2) Constellation diagrams. (a) 4QAM with maximum swing voltage. (b) 16QAM with maximum swing voltage. (c) 16QAM with smaller swing voltage operating in the quasi-linear region. (d) Pre-distorted 16QAM with maximum swing voltage.

For higher-order QAM modulation formats, however, the sinusoidal transfer characteristics of the IQ modulator would 
generate an undesired non-linear distortion on the output signal if no pre-distortion on the input electrical signals is added and the driving peak-to-peak voltages were $2 V_{\pi}$, as for the $4 \mathrm{QAM}$ generation. Fig. 2(b) show the electrical field transfer function and the resultant constellation for a 16QAM signal under these conditions.

Two solutions are typically used to avoid this non-linear distortion in real-time traffic. The first approach relies on generating the components with low peak-to-peak voltages, so the modulator would be operating in the quasi-linear region. Fig. 2(a3-b3) show an example of this solution. The second solution is to digitally pre-distort the driving signals in the sense that the input signals revert the sinusoidal transfer function of the optical modulator (Fig. 2(a4-b4)). This is achievable by digitally processing the electrical signal to be transmitted with an arcsine function.

To generate a dual polarization signal, an integrated polarization diversity modulator based on two IQ modulators and a polarization rotation is typically used. Each of the internal IQ modulator has its bias voltage inputs to control them. In this paper, we distinguish between the bias voltages and the signals, in relation to each of the IQ modulator by adding the indexes $x$ and $y$ to indicate which are related to horizontal (X) and vertical (Y) polarizations, respectively. So, for a dual polarization high-order QAM signal, linearized by either predistorting the electrical input signal or operating it in the quasilinear region,

$$
\begin{aligned}
& E_{\text {out }}(t) \propto e^{j \phi(t)}\left\{\left[s_{i x}(t)+j s_{q x}(t)\right] \vec{X}+\right. \\
& {\left.\left[s_{i y}(t)+j s_{q y}(t)\right] \vec{Y}\right\} . }
\end{aligned}
$$

where $\vec{X}$ and $\vec{Y}$ are the orthogonal polarization direction vectors.

\section{B. Time skew effect in $Q A M$ signals}

Time skew is the delay between two components of a signal. In high-order QAM transmitters, the time skew between the in-phase and quadrature components of each polarization is often referred as IQ time skew, while the time skew between orthogonal polarizations is sometimes referred as $\mathrm{XY}$ time skew [11]. The common cause of time skew are mismatches in the length of the electrical paths in the transmitters. Although a $\mathrm{XY}$ time skews is generally harmless to the signal, since it would sum-up with polarization mode dispersion and be compensated transparently by the receiver DSP, IQ time skews may degrade the received constellation increasing its bit error rate.

Assuming that the in-phase and quadrature components of a single polarization optical signal are time-skewed, then

$$
E_{\text {out }}(t) \propto e^{j \phi(t)}\left[s_{i}(t)+j s_{q}(t-\tau)\right],
$$

where $\tau$ is the time skew between the in-phase and quadrature components. As an example, Fig. 3 shows power eye and constellation/transition diagrams for a few selected values of time skew in a single-polarization 4QAM with non-return-tozero (NRZ) pulse shaping.
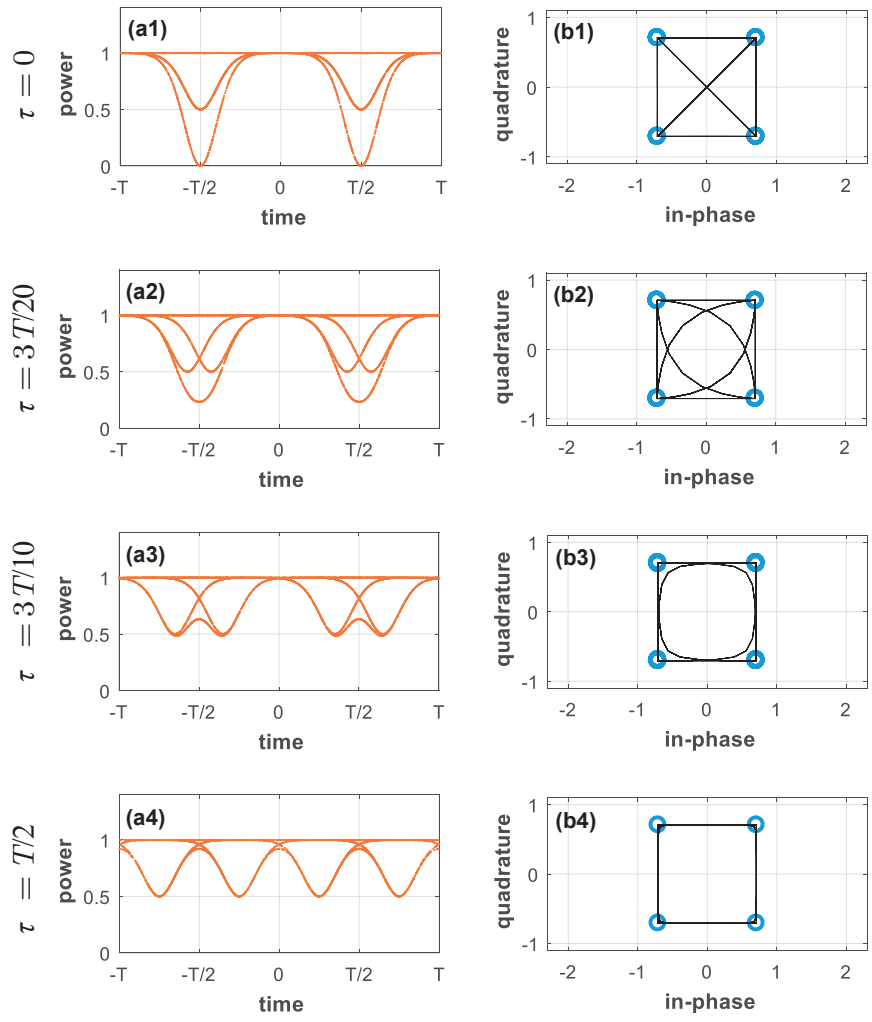

Figure 3. Illustration of the IQ time skew effects in a single-polarization NRZ 4QAM signal. (a) Power eye diagrams. (b) Constellation diagrams (blue circles) and symbol transition paths (black lines). (1) No time skew. (2) Time skew equals to $15 \%$ of symbol period. (3) Time skew equals to $30 \%$ of symbol period. (4) Time skew equals to half symbol period.

One can note in Fig. 3 that while increasing the time skew, the transitions between consecutive symbols tend to cross more distantly from the zero. This can be harmful since the clock tone used for timing synchronization in the receiver indirectly depends on these transitions [10]. In this way, it is possible to use the clock tone information as an effective error function, in order to find the pre-compensation time skew values that would maximize the clock tone.

It has been demonstrated that the clock tone characteristics can be extracted in a directly-detected high-order QAM signal [15]. Hence, passing the time-skewed signal through a photodetector to avoid interference of the laser phase noise, and neglecting the responsivity of the photodetector, the output current, $i(t)$, can be written as

$$
i(t) \propto\left[\left|s_{i}(t)\right|^{2}+\left|s_{q}(t-\tau)\right|^{2}\right],
$$

The directly-detected signal in Eq. 5 is proportional to the sum of the powers of each of the components of the optical signal, i.e., the in-phase and quadrature components.

The clock tone amplitude (CTA) is the maximum value of the timing error detector characteristics and it can be computed for the photodetector output current by [16]

$$
C T A=\left|\sum_{k=1}^{L / S} I(k) I^{*}(k+L-L / S)\right|,
$$

where $I(k)$ is the $L$-sized discrete Fourier transform of the received photocurrent, $i(t)$ (Eq. 5), at $S \geq 2$ samples per 
symbol. The CTA of the photodetected current is proportional to the sum of the clock tones from each of the components of the optical signal. Ideally, the relation between CTA and the time skew, $\tau$, normalized by the maximum possible CTA, is

$$
\frac{C T A(\tau)}{\max (C T A(\tau))}=\left|\frac{1}{2}+\frac{1}{2} \exp \left(j \frac{\tau}{T}\right)\right|,
$$

where $T$ is the symbol period. This relation is illustrated in Fig. 4. The CTA will have its maximum values for time skew $\tau=n T, \forall n \in \mathbb{Z}$, and it will have its minimum values for time skew $\tau=(n+1 / 2) T, \forall n \in \mathbb{Z}$. So, a time skew estimator based on CTA maximization will have its estimation range limited by the interval $-T / 2<\tau<T / 2$.

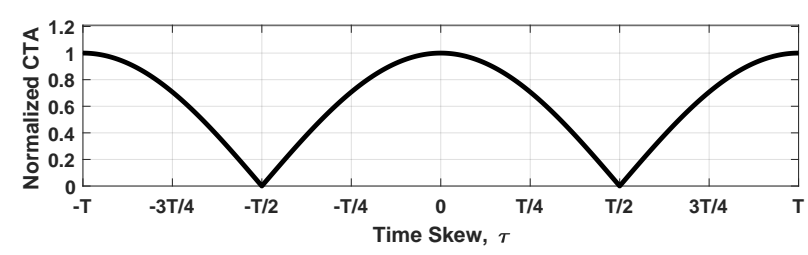

Figure 4. Theoretical curve of CTA relative to transmitter time skew in a single-polarization signal.

Analogously, for a time-skewed dual-polarization signal, the field can be written as

$$
\begin{aligned}
E_{\text {out }}(t) \propto & e^{j \phi(t)}\left\{\left[s_{i x}(t)+j s_{q x}\left(t-\tau_{x}\right)\right] \vec{X}\right. \\
& \left.+\left[s_{i y}\left(t-\tau_{x y}\right)+j s_{q y}\left(t-\tau_{x y}-\tau_{y}\right)\right] \vec{Y}\right\},
\end{aligned}
$$

where $\tau_{x}$ is the time skew between IQ components of polarization $\mathrm{X}, \tau_{y}$ is the time skew between IQ components of polarization $\mathrm{Y}$, and $\tau_{x y}$ is the time skew between polarizations. Rewriting the time skews as the time skews relative to the inphase component of polarization $\mathrm{X}, \tau_{1}=\tau_{x}, \tau_{2}=\tau_{x y}$, and $\tau_{3}=\tau_{x y}+\tau_{y}$, the photocurrent generated after passing the dual-polarization signal through a photodetector is

$$
\begin{aligned}
i(t) \propto\left[\left|s_{i x}(t)\right|^{2}+\mid\right. & \left.s_{q x}\left(t-\tau_{1}\right)\right|^{2}+ \\
& \left.\left|s_{i y}\left(t-\tau_{2}\right)\right|^{2}+\left|s_{q y}\left(t-\tau_{3}\right)\right|^{2}\right] .
\end{aligned}
$$

The CTA behaves in a similar way compared to the singlepolarization case, as illustrated in Fig. 5. The maximum CTA will only be achieved when all the values of the time skews are equal to zero, as shown in Fig. 5(a).
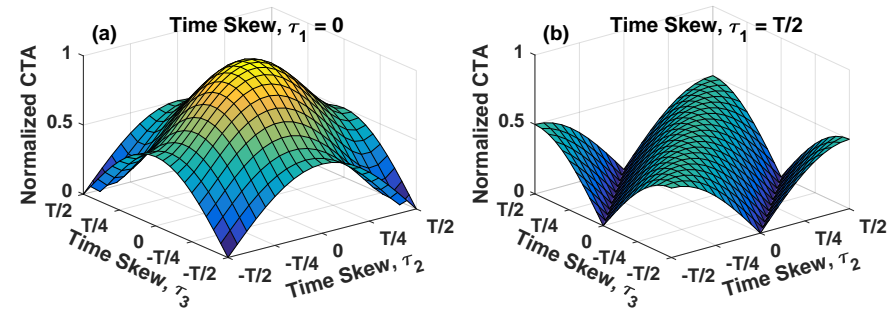

Figure 5. Theoretical curves of CTA relative to transmitter time skew in a dual-polarization signal varying two values of time skews, while maintaining the remaining constant. (a) $\tau_{1}=0$; (b) $\tau_{1}=\mathrm{T} / 2$.

\section{QAM signal with improper biasing}

1) Operation points: The children bias voltages move the center location of the signal constellation in a non-linear way due to the sinusoidal transfer function of the IQ modulator. For small peak-to-peak swing voltages, children bias voltages different from the optimum value, $-V_{\pi}$, will change not only the constellation points but also the symbol transition paths, as can be seen in Fig. 6(c). The transition paths will cross more distantly from the zero and then, analogously to the time skew case, reduce the CTA absolute value. This bias-dependent behavior of the CTA can be explored for the calibration of optimum values for the children bias voltages.
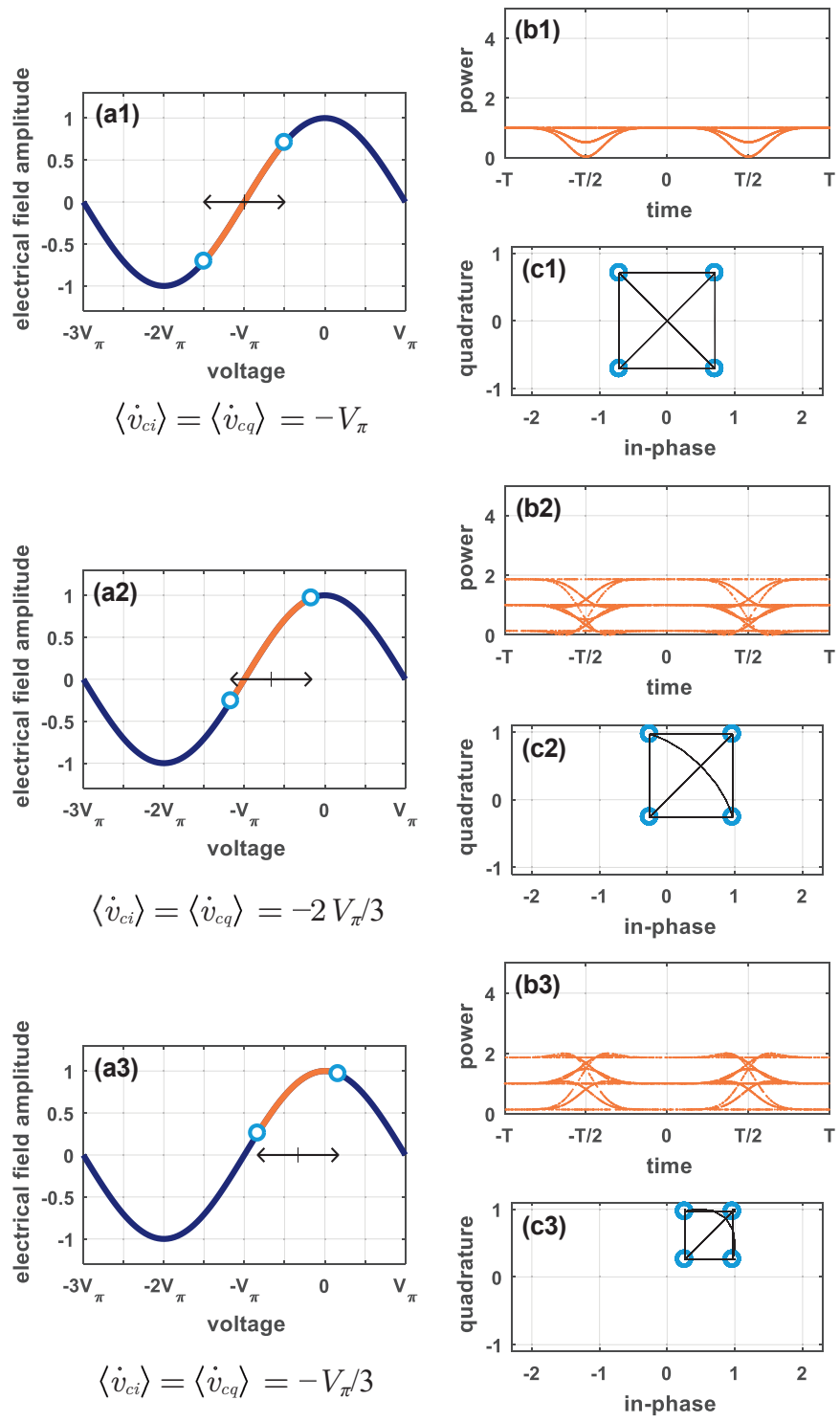

Figure 6. Illustration of different children bias operation points in a singlepolarization NRZ 4QAM signal. (a) Electrical field transfer functions. (b) Power eye diagrams. (c) Constellation diagrams (blue circles) and symbol transition paths (black lines). (1) $-V_{\pi}$ children bias. (2) $-2 V_{\pi} / 3$ children bias. (3) $-V_{\pi} / 3$ children bias.

However, variations in the children bias voltages change the power of the output optical signal, affecting in a different fashion the CTA. To avoid interference from the power, we 
introduce the modified clock tone amplitude (MCTA),

$$
\text { MCTA }=\frac{\left|\sum_{k=1}^{L / S}\left[I(k) I^{*}(k+L-L / S)\right]\right|}{\sum_{k=1}^{L}\left[I(k) I^{*}(k)\right]} .
$$

Fig. 7 shows the MCTA behavior for different values of the parent bias voltage, while varying the children bias voltages for a single polarization signal. The MCTA have its maximum value when the children bias voltages are optimum, and maintain the concave shape for different values of the parent bias voltages, being robust to its variation. For the dual polarization case, the MCTA behaves analogously, having its global maximum values for the optimum values of all four children bias voltages.

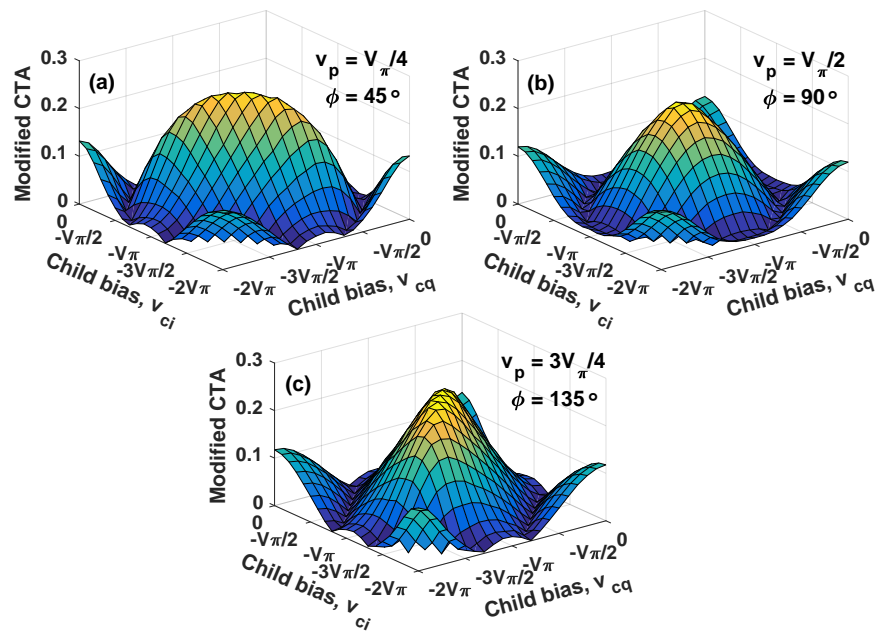

Figure 7. Theoretical curves of MCTA relative to children bias voltages in a single-polarization NRZ 4QAM signal with $4 V_{\pi} / 5$ peak-to-peak swing voltages. Parent bias: (a) $V_{\pi} / 4$. (b) $V_{\pi} / 2$. (c) $3 V_{\pi} / 4$.

2) IQ phase: The parent bias voltages are responsible for the adjustment of the constellation phases in each polarization. In Fig. 8, one can notice that the zero crossings are independent of the parent bias voltage. Thus, in order to find the optimum values for these voltages, the CTA is not a suitable metric. However, another statistical property of the optical power signal can be used. It is clear by Fig. 8 that when $v_{P}$ is correctly adjusted to $V_{\pi} / 2$, i.e., perfect orthogonality between in-phase and quadrature components, the optical power of all symbols are equal. Meanwhile, if the parent bias voltage moves away from its optimum value, the power corresponding to each of the symbols disperse, increasing the power variance. This way, it is possible to use the variance of the optical power signal, i.e., the variance of the photodetected signal, as an indicator of the optimum value for the parent bias voltage. So, if the children bias are correctly set, the variance will be minimized when the parent bias is in its optimum value. Alternatively, one could maximized the inverse of the variance to find the optimum parent bias value.

Fig. 9(a) and Fig. 9(c) show the variance versus parent bias voltage curve for a dual polarization signal with optimum children bias voltages, with 4QAM and 16QAM, respectively.
Fig. 9(b) and Fig. 9(d) show the inverse of the variance for the same cases. The inverse of the variance can be used as an alternative fitness function to be maximized. It is also important to note that the variance curve have similar shape also for QAM modulation formats of different orders.
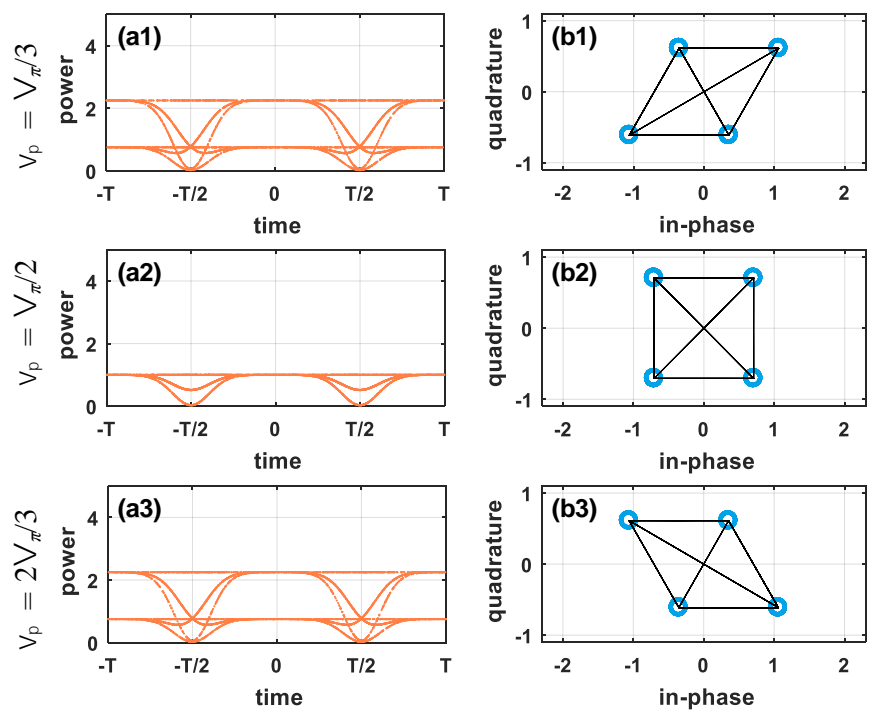

Figure 8. Illustration of different parent bias in a single-polarization NRZ 4QAM signal. (a) Power eye diagrams. (b) Constellation diagrams (blue circles) and symbol transition paths (black lines). (1) $V_{\pi} / 3$ parent bias. (2) $V_{\pi} / 2$ parent bias. (3) $2 V_{\pi} / 3$ parent bias.
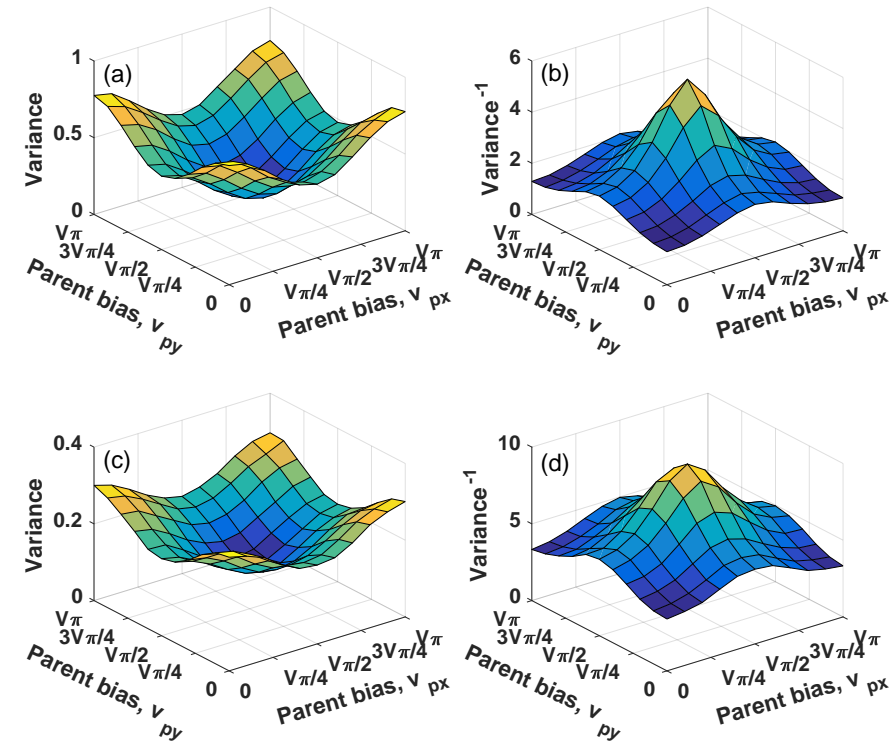

Figure 9. Theoretical curve from the photodetected signal relative to parent bias voltages in a dual-polarization signal with $4 V_{\pi} / 5$ peak-to-peak swing voltages and optimum children bias voltages. (a) Variance for NRZ 4QAM. (b) Inverse of variance for NRZ 4QAM. (c) Variance for NRZ 16QAM. (d) Inverse of variance for NRZ 16QAM.

\section{Amplitude mismatch in QAM signals}

An amplitude mismatch between components of the optical signal may arise at the transmitter due to different attenuation in electrical paths and mismatched gains in electrical driver 
amplifiers. For a transmitter without time skew, operating at optimum bias voltages for QAM transmission and swing voltages inside the linear region, the amplitude mismatched dual-polarization optical signal can be written as

$$
\begin{array}{r}
E_{\text {out }}(t)=e^{j \phi(t)}\left\{\left[A_{\text {ix }} s_{i x}(t)+j A_{q x} s_{q x}(t)\right] \vec{X}+\right. \\
\left.\left[A_{i y} s_{i y}(t)+j A_{q y} s_{q y}(t)\right] \vec{Y}\right\},
\end{array}
$$

where $A_{i x}, A_{q x}, A_{i y}$, and $A_{q y}$ are the amplitudes for each of the signal components, and $s_{i x}(t), s_{q x}(t), s_{i y}(t)$, and $s_{q y}(t)$ have unitary maximum amplitude.

An iterative method to estimate and compensate for these amplitude imbalances can be applied. It starts by defining amplitude imbalance correction factors, $C_{q x}, C_{i y}$, and $C_{q y}$, for each of the components, except the in-phase component from the polarization $X$, that is used as a reference value. These correction factors are multiplied by the signal components in the digital domain, before digital-to-analog conversion, such that the output signal is approximated by

$$
\begin{array}{r}
E_{\text {out }}(t)=e^{j \phi(t)}\left\{\left[A_{i x} s_{i x}(t)+j C_{q x} A_{q x} s_{q x}(t)\right] \vec{X}+\right. \\
\left.\left[C_{i y} A_{i y} s_{i y}(t)+j C_{q y} A_{q y} s_{q y}(t)\right] \vec{Y}\right\},
\end{array}
$$

A signal only with the in-phase component of one of the polarizations is then generated by "turning off" the other components, i.e., reducing their swing voltages to zero. This turn-off procedure is possible only if the modulator is correctly biased for a QAM transmission, so a zero voltage on the input would result in minimum power on the output. Then, this single component signal is photodetected and sampled, and its power is computed by

$$
P_{i x}=\sum_{k=1}^{N} i(k),
$$

where $i(k)$ is the sampled photodetected current, and $N$ is the number of samples acquired. Then, the power of each of the other components is computed. The correction factor for the amplitude imbalance is updated by

$$
C_{h, \text { new }}=C_{h, \text { old }} \sqrt{\frac{P_{i x}}{P_{h}}}, h \in\{q x, i y, q y\} .
$$

Due to the sinusoidal response of the modulators, these new correction factors are not immediately the best values, needing some iterations to converge to optimum values. After iterating the algorithm, the correction factors will converge to assure that $A_{i x}=C_{q x} A_{q x}=C_{i y} A_{i y}=C_{q y} A_{q y}$.

\section{GA-BASED METHOd FOR TRANSMITTER PARAMETERS CALIBRATION}

To estimate and compensate for the dual polarization transmitter front-end imperfections presented in the last section, it is possible to use the information extracted from the directly detected signal to optimize the aforementioned transmitter using a genetic algorithm and a turn-on/turn-off procedure. In this section the genetic algorithm is discussed and then the proposed method for transmitter optimization is introduced.

\section{A. Genetic algorithm for parameters optimization}

Genetic algorithm (GA) is a particular class of evolutionary algorithms that has been successfully used to optimize a great variety of problems [17]-[20]. A typical genetic algorithm uses techniques inspired by evolutionary biology, as heredity, mutation, natural selection and crossover, being notably efficient to find good solutions in problems with many variables, and in the presence of noise. Thus, the GA is a suitable solution for transmitter parameters optimization, due to the quantity of variables to optimize and the noise present in CTA, MCTA and variance extraction processes. Additionally, it enables avoiding local extrema (minima or maxima).

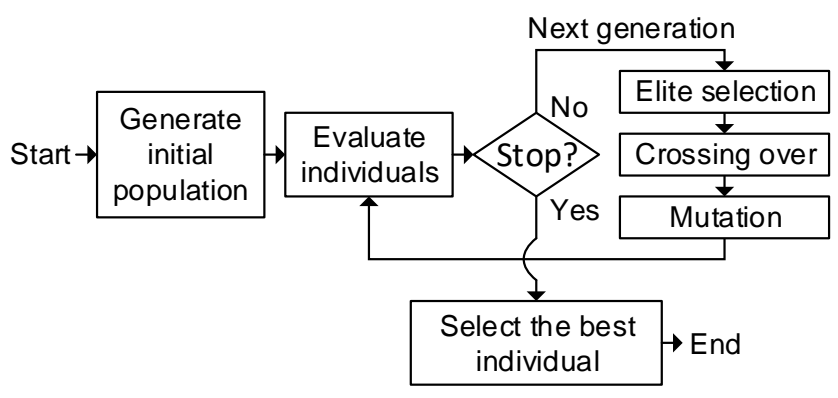

Figure 10. Genetic algorithm block diagram.

The basic implementation of a GA is shown in Fig. 10. The idea behind GAs is to perform optimization of solutions as living beings would evolve in the wild nature through generations. It starts by randomly creating a set of starting solutions. These solutions are treated as individuals of a population and the variables of the solutions are their chromosomes. Each of the individuals are evaluated through a fitness function and then genetic operations are made. The weakest individuals die and the strongest individuals are labeled as the elite group. A new offspring is then produced composed of a pure copy of the elite group, crossover from pairs of elite chromosomes and mutations based on the elite group. A new generation is then started and the process continues being repeated until a stopping criteria is met. This stopping criteria can be when the improvement from successive generations is negligible or, more commonly, when a certain generation is reached.

Tab. I shows a list of parameters of the dual polarization optical modulator along with the information that can be extracted from the the directly detected signal and be used as fitness functions for optimization.

Table I

List of PARAmeters Along Fitness Functions

\begin{tabular}{lll}
\hline Parameter & Variables & Fitness function \\
\hline Time skew & $\tau_{1}, \tau_{2}, \tau_{3}$ & CTA \\
Child bias voltage & $\dot{v}_{c i x}, \dot{v}_{c q x}, \dot{v}_{c i y}, \dot{v}_{c q y}$ & MCTA \\
Parent bias voltage & $v_{p x}, v_{p y}$ & Variance \\
Amplitude mismatch & $C_{q x}, C_{i y}, C_{q y}$ & $\begin{array}{l}\text { Power per quadrature } \\
\text { and pol. component }\end{array}$ \\
\hline
\end{tabular}

Then, as the transmitter impairment optimization is a problem with multiple fitness functions a multi-objective genetic 
algorithm (MO-GA) seems appropriate [20]. However, multiple solutions for a single problem could exist leading to ambiguities and sub-optimal solutions. By the other hand, a cooperative coevolutionary approach of genetic algorithms (CC-GA) can take advantage of the partial independence among the fitness functions and ensure a faster and correct convergence to an optimum solution [21].

The CC-GA divides a larger population into subpopulations, solving them sequentially and iteratively. In this case, each of the subpopulations is called species and an individual from a species is called specimen. A specimen's chromosome is constituted by a subset of the variables from the larger population. The only interaction between species is in the cooperative evaluation of each specimen, when the specimens being evaluated are combined with specimens randomly selected from the other species' elite group. A full chromosome with all the parameters is formed by the combination of one specimen of each species. This way, the CC-GA simulates the cooperative evolution in the wild nature among different species.

In the case of transmitter parameters optimization, the larger problem can be reduced into three different species: the time skews $\left(\tau_{1}, \tau_{2}, \tau_{3}\right)$, the children bias voltages $\left(\dot{v}_{c i x}, \dot{v}_{c q x}, \dot{v}_{c i y}\right.$, $\left.\dot{v}_{c q y}\right)$, and the parent bias voltages $\left(v_{p x}, v_{p y}\right)$. The fitness functions from each of the species are the CTA, MCTA, and the variance of the photodetected current, respectively. Fig. 11 shows a block diagram of the CC-GA implementation. It starts randomly creating a population for each of the species. The data processing is done for one species at a time. For the first species, each specimen is randomly associated with one specimen from each of the other species elite group, e.g., if time skews are the first species, so each time skews' specimen will be associated to one elite children bias voltages' specimen and one elite parent bias voltages' specimen. These cooperative combinations are evaluated though the first fitness function, and genetic operations of elite selection, crossover and mutation are performed. It is important to note that a one-to-one mapping is not necessary, so one specimen from a different species' elite group can be associated with more than one specimens of the species being evaluated. The elite group for the first species is then updated and the process is repeated regarding the next species. After the last species evaluation, a new offspring is generated and then the whole process is repeated until a stopping criteria is met. At that point the best specimen of each species is selected, forming the final solution.

\section{B. Proposed method: $C C-G A+$ turn-on/turn-off procedure}

A $M$-QAM reference signal is generated in the transmitter and used to estimate the transmitter impairments. This reference signal should be a dual polarization signal operating in the quasi-linear region of the modulator, and should have a certain pulse-shape, modulation format and symbol rate. To avoid the influence of transmitter laser phase noise and coherent receiver impairments, the signal is detected by employing direct detection. The output photocurrent is sampled in an analog-to-digital-converter (ADC) with a sampling frequency greater than twice the symbol rate being used.

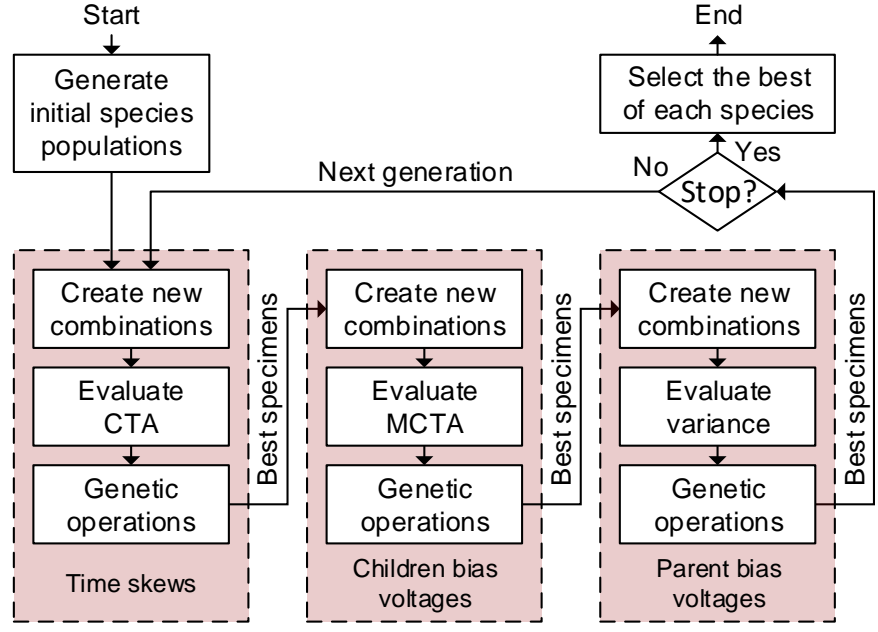

Figure 11. Cooperative coevolutionary genetic algorithm block diagram.

Next, the fitness functions are computed from the sampled signal. These fitness functions are fed to the CC-GA that iteratively controls the bias voltages and time skews by evaluating the associated fitness functions. Between each CC-GA generation, the best time skews and bias voltages are set and an iteration of a turn-on/turn-off procedure as presented in Section II.D is done for amplitude mismatch estimation and compensation. When a stopping criteria is met, the best solution is then selected as the final calibration parameters. Fig. 12 shows a schematic for the transmitter front-end imperfections calibration. After the calibration ends, as all the transmitter parameters would be optimized, it is possible to change to a different pulse shape, modulation format and symbol rate, as well as to use pre-distortion.

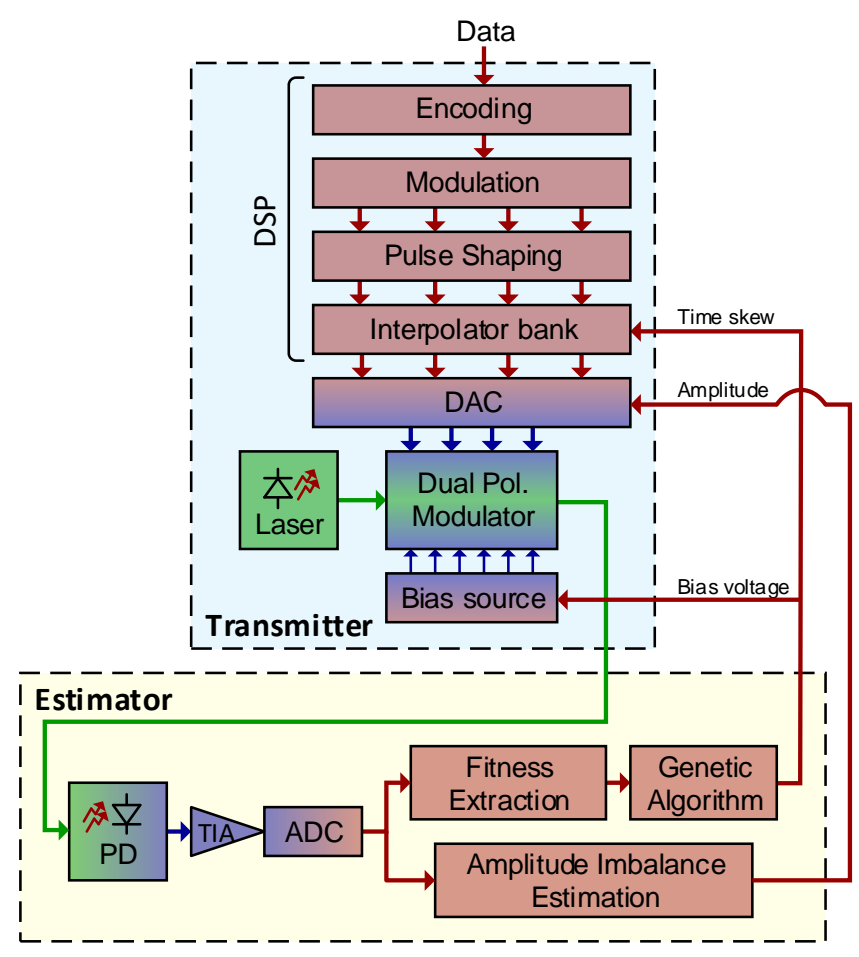

Figure 12. Proposed algorithm scheme. 


\section{RESUlts AND ANALYSis}

\section{A. Simulation Analysis}

In order to evaluate the performance of the proposed method, the simulation setup of Fig. 13 was used. First, sequences of bits are generated at a pseudo random bit sequence (PRBS) generator with length 31 . These sequences are mapped to a modulation format at 1 sample per symbol and then filtered with a raised cosine pulse shape at 2 samples per symbol and roll-off 1, emulating an NRZ pulse shaping. The signal is then quantized with 8-bit resolution to emulate the limitations of a digital to analog converter. The signal is then resampled to a new sampling rate with each of the signal components being time delayed in order to account for the time skews. The signal components are then low-pass filtered and have their peak-to-peak values adjusted, emulating an electrical driver. The components are finally fed to a dual polarization modulator, with variable bias voltages. Noise is added to the signal, which is then received in a photodiode. The cost functions are computed from the photodetected signal and fed to a parameter controller that will control the time skew, amplitude and bias voltage values accordingly to the proposed method.

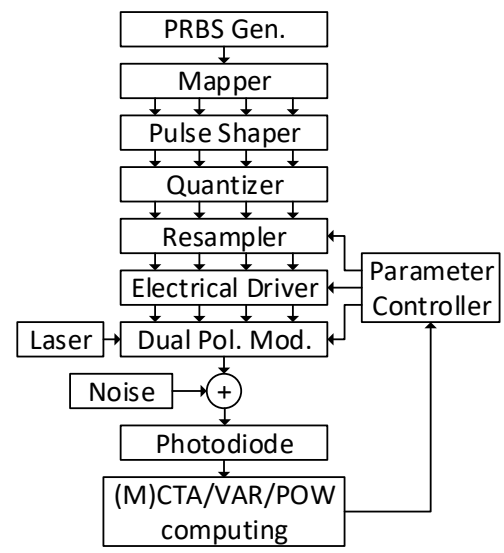

Figure 13. Simulation setup for transmitter optimization evaluation.

Unless stated otherwise, all the simulation results were extracted by the aforementioned setup with a dual polarization NRZ 16QAM reference signal operating at $16 \mathrm{GBd}$. The quantity of symbols used for each fitness function computation was 16384. The optical modulator was an ideal dual polarization modulator with $V_{\pi}=4 \mathrm{~V}$, and no additional time skew. The reference peak-to-peak input signal voltages were selected to be $1.6 \mathrm{~V}$, in order to operate inside the quasi-linear region of the optical modulator when correctly biased. Noise was added to the signal to guarantee an $18 \mathrm{~dB}$ OSNR $(0.1 \mathrm{~nm}$ resolution) at the output of the modulator. The number of generations considered for the genetic algorithm was 50, and the time skew, child bias voltage, and parent bias voltage populations were 60,80 , and 50 specimens, respectively, in which, after each generation, $40 \%$ of the specimens were selected as elite, $50 \%$ mutated, and $10 \%$ passed through a crossing-over process. The number of simulation runs to compute the accuracy of the method was 100 .
First, to assess the convergence speed of our proposed method we ran it with the simulation parameters stated above. The estimated parameters after each generation are depicted in Fig. 14. These estimated values are the average of the values of all specimens selected as elite. The expected values for this simulation were $-4 \mathrm{~V}$ for the children bias voltages (equivalent to $\left.-V_{\pi}\right), 90^{\circ}$ for the IQ phase, 0 for the time skews and 1 for the relative amplitudes.
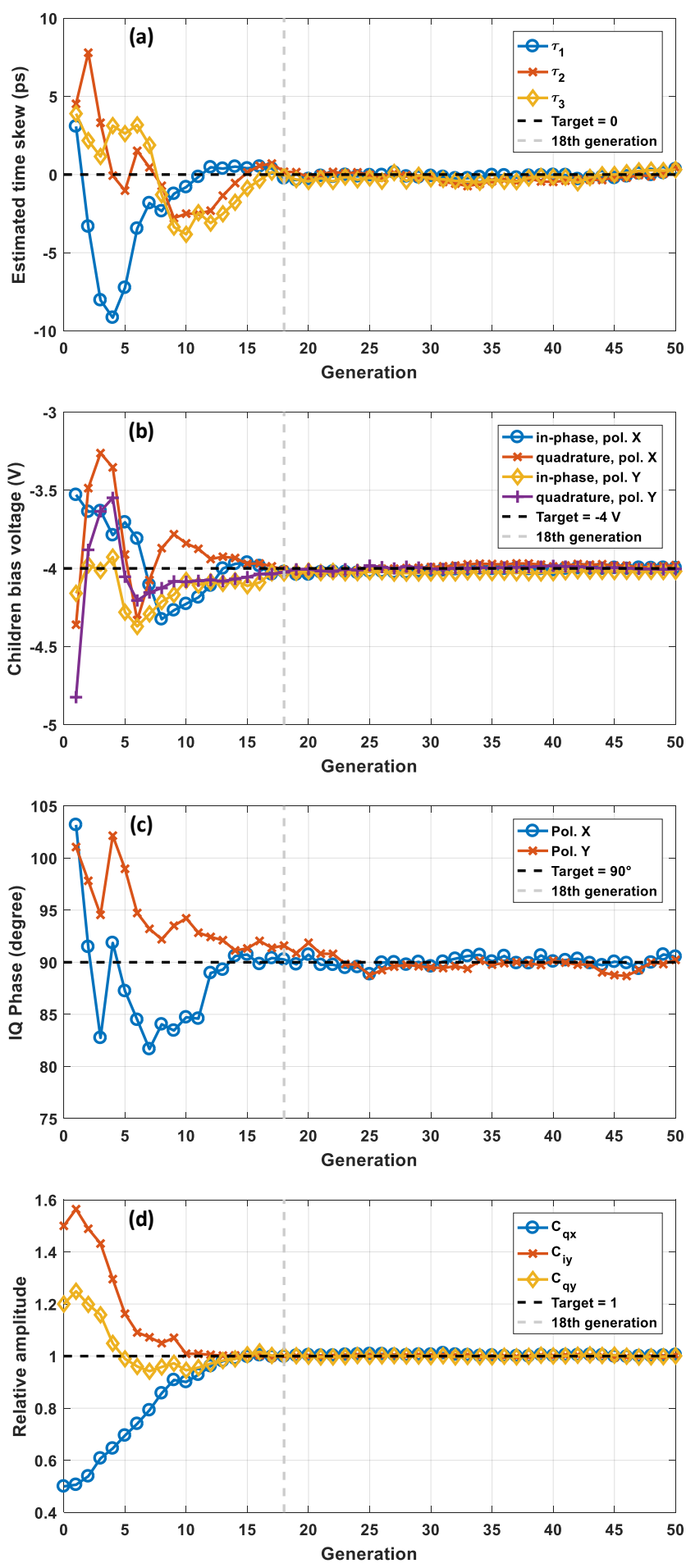

Figure 14. Evolution through generations of the estimated values. (a) Time skew. (b) Children bias voltage. (c) Phase between in-phase and quadrature components. (d) Amplitude relative to the base component. 
We can see in Fig. 14 that after the 18th generation all values seem to have converged to their expected value. This means that 3420 fitness-functions calculations and 54 power amplitude measurements were needed to converge to the final estimated values. Thus, the total calibration time would be limited by the amount of time needed for the IQ modulator stabilization and the optimum population sizes for the proposed algorithm. Nevertheless, if compared with full BER-based optimization, our proposed method is believed to significantly reduce calibration time since it significantly reduces the required data processing.

Another approach for convergence verification is to evaluate the evolution of the fitness values used at the CC-GA algorithm. The aforementioned evolution is depicted in Fig. 15.
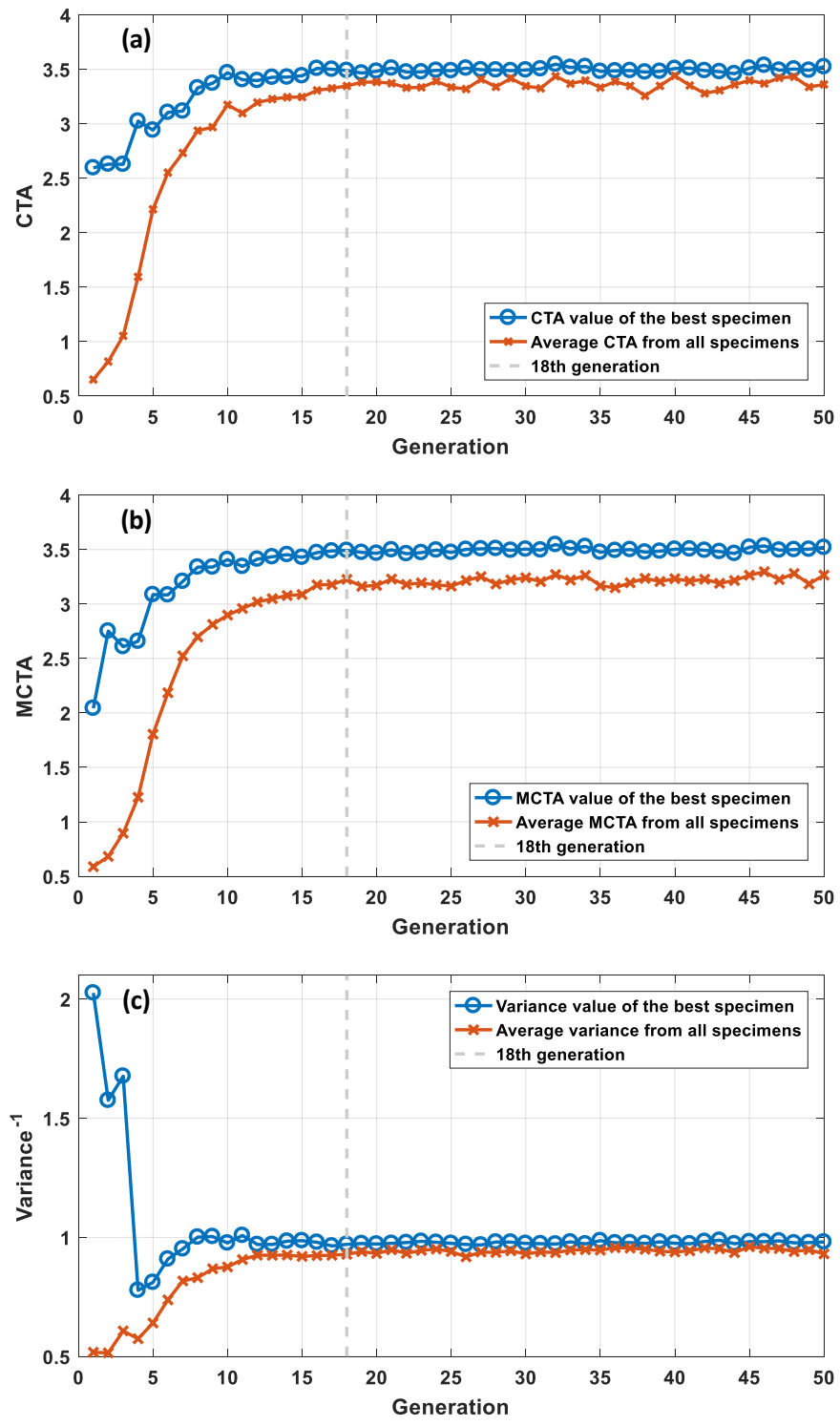

Figure 15. Evolution through generations of the fitness values. (a) Clock tone amplitude. (b) Modified clock tone amplitude. (c) Inverse of the variance.

When the average of the fitness values from all the specimens approximate the fitness value obtained by the best specimen it means that all specimens are close to the optimal solution, therefore, converged. A stopping criteria may be set when the average fitness function of all specimens exceeds a percentage of the fitness value of the best specimen. In this case, the stopping criteria would be $95.1 \%, 92.3 \%$, and $95.5 \%$ for the CTA, MCTA and inverse variance fitness functions.

To evaluate the proposed method performance we measured the accuracy and precision of the method through Monte Carlo simulations. The accuracy and the precision were assessed by the absolute mean estimation error and the standard deviation relative to the target values, respectively. First, we analyzed the performance using reference signals with different modulation formats, running the proposed method 100 times, and then analyzing the last 10 generations from each of the iterations. This way, the data size used to compute the mean and standard deviation was 1000. The results are shown in Fig. 16 for the absolute mean estimation error, and in Fig. 17 for the standard deviation.

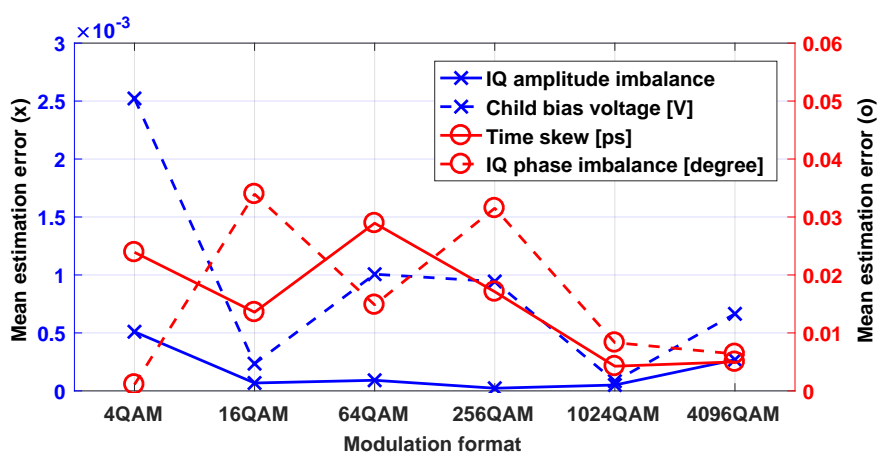

Figure 16. Absolute mean estimation error compared to target values for different modulation formats.

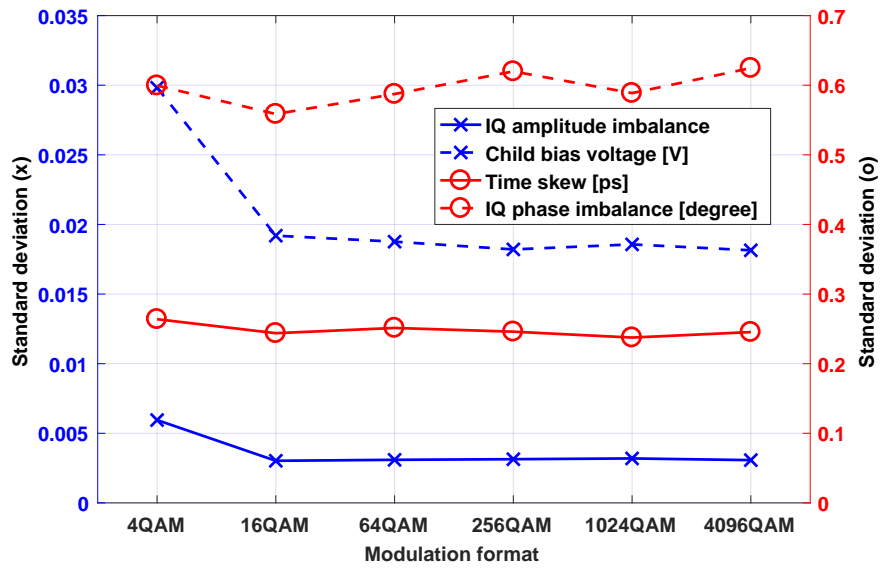

Figure 17. Standard deviations for different modulation formats.

The the standard deviations are one order of magnitude larger than the mean estimation error. This means that the method can be considered very accurate, as the errors in the estimation process are more random than systematic. The best reference signal in this case was the NRZ 16QAM that had standard deviations of $0.56^{\circ}$ for the IQ phases, $0.019 \mathrm{~V}$ for the children bias voltages, $0.24 \mathrm{ps}$ for the time skews and 0.003 for the amplitude imbalance. The distribution of the estimated values was Gaussian shaped, meaning that $99.7 \%$ 
of the estimations are expected to fall inside an interval of 3 times the standard deviation.

The proposed method has also been characterized testing the number of symbols used for each fitness function calculation. The results are shown in Fig. 18 and Fig. 19.

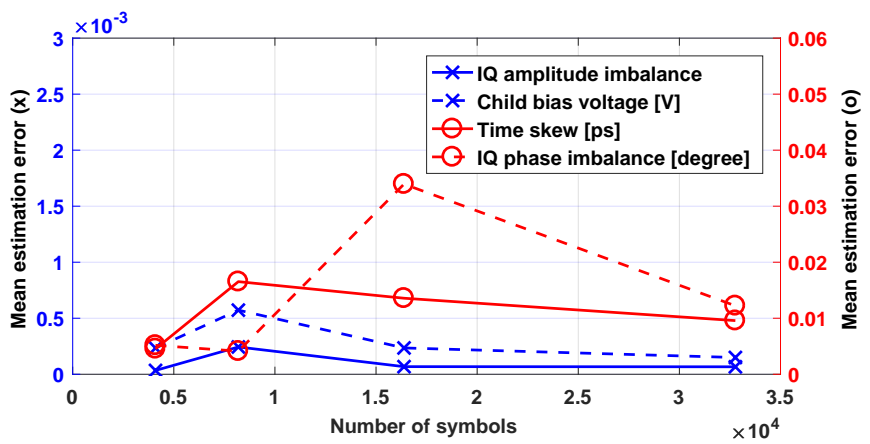

Figure 18. Absolute mean estimation error compared to target values for different number of symbols for each cost-function calculation with NRZ 16QAM reference signal.

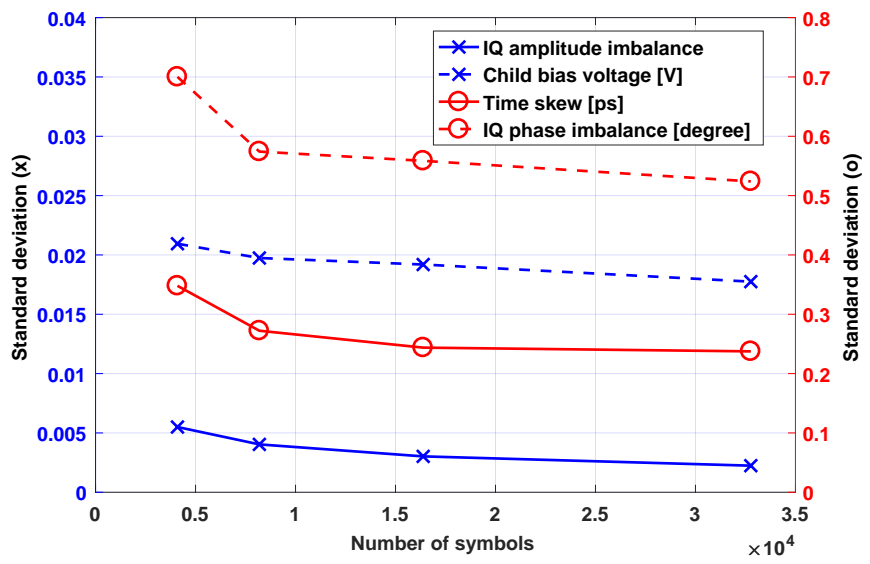

Figure 19. Standard deviations for different number of symbols for each cost-function calculation with NRZ 16QAM reference signal.

Again, the standard deviations are one order of magnitude larger than the mean estimation error. Increasing the number of symbols used to compute every fitness value will increase the precision of the method. The number of symbols used in each fitness function computation will influence how fast the method convergence speed can be. The results show that doubling the number of symbols from 65536 to 131072 has a higher impact than doubling further on.

As a performance metric we analyzed the penalty in the OSNR required to achieve a given bit error rate (BER) due to each of the transmitter impairments. Signals operating at $16 \mathrm{GBd}$ were generated with 4QAM, 16QAM and 64QAM as modulation formats and then received in a common DSP-based dual polarization coherent receiver with decisiondirected least-mean-square MIMO equalization, blind phase search carrier recovery, and standard decision regions for bit demapping [3]. The OSNR penalty was numerically measured when the signals were transmitted through an additive white Gaussian noise channel by varying the noise parameter. The results are shown in Fig. 20.
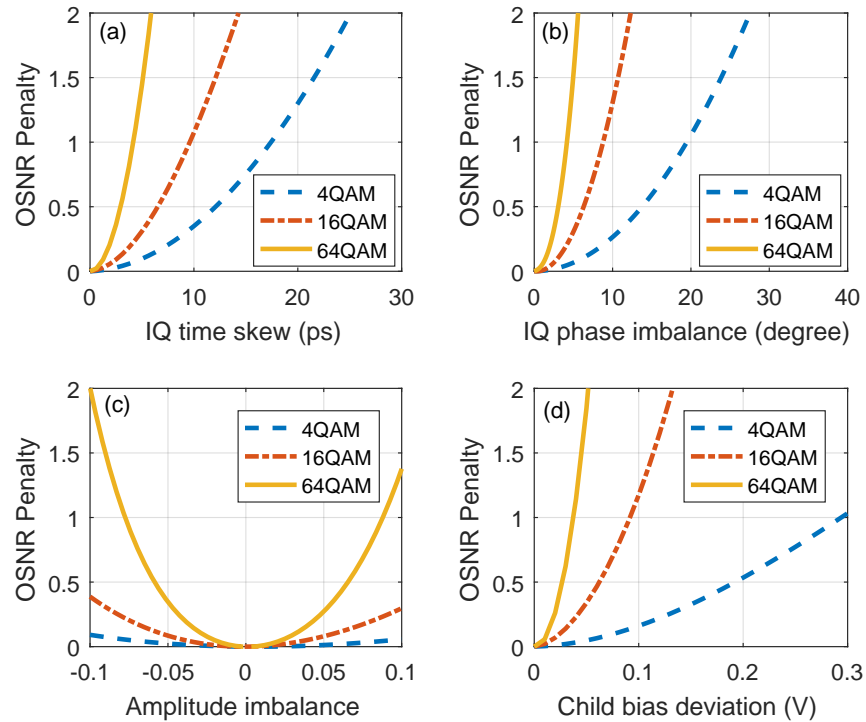

Figure 20. OSNR penalty for a 16-GBd signal, $V_{\pi}=4 \mathrm{~V}$, and peak to peak voltages of $1.6 \mathrm{~V}$ at BER $=3.8 \times 10^{-3}$ for 4QAM and 16QAM and at $\mathrm{BER}=10^{-2}$ for 64QAM, due to: (a) IQ time skews; (b) IQ phase imbalance (c) Amplitude imbalance; (d) Child bias voltage error.

Then, considering the worst case scenario as each of the transmitter impairments calibrated with an error of 3 times the standard deviation, we numerically measured the OSNR penalties at BER $=3.8 \times 10^{-3}$ for the QPSK and the 16QAM signals as $0.05 \mathrm{~dB}$ and $0.5 \mathrm{~dB}$, respectively. For the 64QAM signal a $1.9 \mathrm{~dB}$ OSNR penalty at $\mathrm{BER}=10^{-2}$ was measured. These penalties were mainly due to the child bias voltages and could be drastically reduced to $0.05 \mathrm{~dB}$ and $0.2 \mathrm{~dB}$ OSNR penalty for the 16QAM and 64QAM modulation formats, respectively, if a simple change in the decision regions at the receiver DSP is considered.

In comparison to other algorithms, IQ time skew can be calibrated using the method presented by Fludger et al. [12] with typical accuracy of $0.5 \mathrm{ps}$. Additionally to the IQ time skews, the method presented by Yue et al. [11] could find also XY time skews in the range of $0.5 \mathrm{ps}$. Our proposed method have similar performance compared to these alternative methods while also calibrating the IQ phase imbalance, the IQ amplitude imbalance, and the bias operation voltages.

\section{B. Experimental Validation of Time Skew Calibration with GA}

In the previous section we had a numerical evaluation of the proposed method. In this subsection and in the next one, we will verify the performance of the GA for time skew estimation, and demonstrate the behavior of the CC-GA for time skew and bias voltage calibration.

To evaluate the CTA behavior, we first tested the modulators separately performing the experiment at single polarization. We generated an NRZ 4QAM signal at $32 \mathrm{GBd}$ and we swept the pre-compensation IQ skew from -24 to 24 ps with steps of 0.1 ps. For each skew value we acquired ten different traces and plotted the average of CTAs for both modulators (Fig. 22).

As expected, the maximum CTAs were at the IQ skew values of $\tau_{x}=-6 \pm 0.05$ ps and $\tau_{y}=-10 \pm 0.05$ ps. One can 


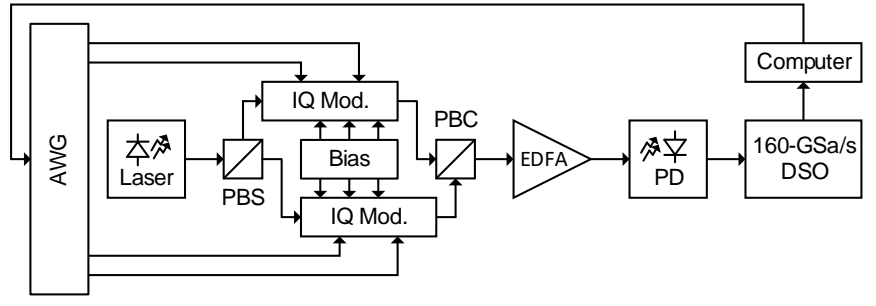

Figure 21. Experimental setup for time skew calibration.

also note that the distance between the two minimums were $31.25 \mathrm{ps}$ which is exactly the symbol period at $32 \mathrm{GBd}$.

Then, we generated a 32-GBd dual-polarization NRZ 4QAM signal. The first time skew, $\tau_{1}$ was used as -6 and 9.125 ps, representing the best and worst case scenario, respectively, and the remaining time skews were swept from -25 to $25 \mathrm{ps}$ with steps of $1.25 \mathrm{ps}$. A subset of the found CTA values are shown in Fig. 23.

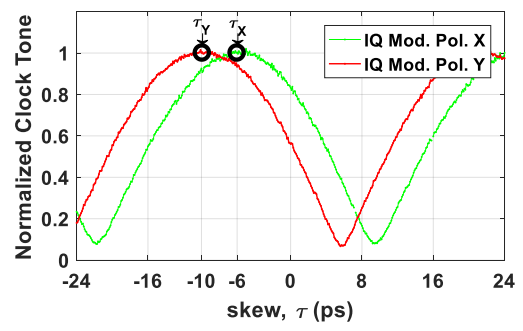

Figure 22. Experimental curves for clock tone relative to transmitter skew in a single polarization signal.

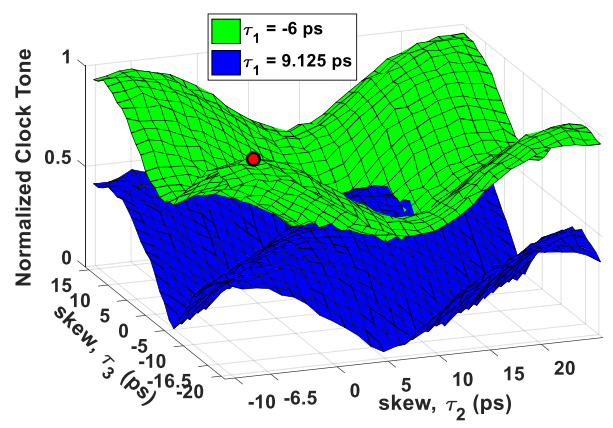

Figure 23. Experimental curves for clock tone relative to transmitter skew in a dual polarization signal.

In this case, the time skew values that maximized CTA were $\tau_{1}=-6 \mathrm{ps}, \tau_{2}=-6.25 \pm 0.625 \mathrm{ps}$, and $\tau_{3}=-16.25 \pm 0.625 \mathrm{ps}$, that are equivalent to the same IQ skews found previously and the time skew between polarizations, $\tau_{x y}=-6.25 \pm 0.625 \mathrm{ps}$.

To evaluate the performance of the GA as searching method for the time skew estimator we created a random population of 50 three-dimensional individuals $\left(\tau_{1}, \tau_{2}, \tau_{3}\right)$, uniformly distributed in the interval between -15 and 15 ps. After each generation the GA selected the 10 individuals associated with the highest CTA values as elite individuals and performed cross-over and mutation on the other individuals based on them. We ran the GA through 35 generations and the evolution of CTA values are shown in Fig. 24.
After the last generation the average of the elite individuals was $\tau_{1}=-6.33 \mathrm{ps}, \tau_{2}=-6.53 \mathrm{ps}$, and $\tau_{3}=-16.46 \mathrm{ps}$, which are consistent with the time skew values previously found. We repeated the GA procedure 5 times and all the resulting skew values found were inside a small interval of $\pm 0.5 \mathrm{ps}$.

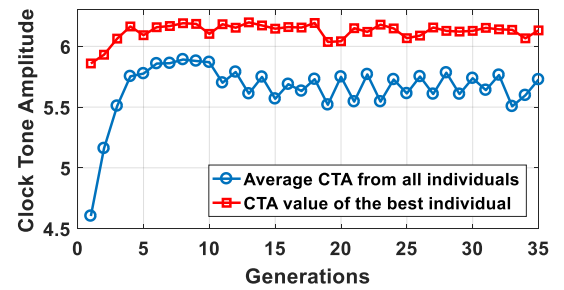

Figure 24. Experimental CTA evolution for a GA-based time skew estimator.

\section{Experimental Demonstration of Time Skew and Operation Point Calibration with $C C$-GA}

Finally, to demonstrate the method behavior an experiment with a dual polarization modulator is reported. Four output channels of a $64-\mathrm{GSa} / \mathrm{s}$ AWG were applied to a dual polarization Mach-Zehnder-based IQ modulator, used to generate a reference signal at 16 GBd and NRZ DP-16QAM modulation format. The generated signal was amplified by an EDFA, directly-detected in a 45-GHz bandwidth PD and then sampled by a DSO operating at $160 \mathrm{GSa} / \mathrm{s}$. The fitness functions for the CC-GA were calculated on a personal computer that was also used to automatically control the time skew pre-compensation values in the AWG and the modulator bias voltages. The time skews and the correct operation points for this setup were previously unknown, with initial voltages being random and not resulting into recoverable constellations. The number of generations considered for the CC-GA was 30 , and the time skew, child bias voltage, and parent bias voltage populations were 60,80 , and 50 specimens, respectively, in which, after each generation, $40 \%$ of the specimens were selected as elite, $50 \%$ mutated, and $10 \%$ passed through a crossing-over process. The experimental setup is shown in Fig. 25.

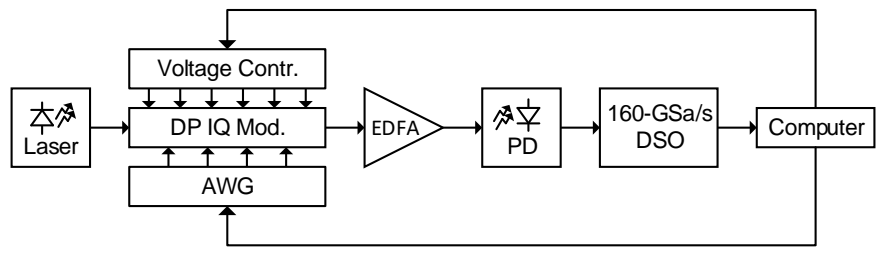

Figure 25. Experimental setup for time skew and operation point calibration.

To assess the convergence we ran the experiment with the parameters stated above. The estimated parameters after each generation are depicted in Fig. 26, while the evolution of the fitness functions is depicted in Fig. 27.

The estimated values are the average of the values of all specimens selected as elite and the fitness values are the CTA, MCTA and inverse variance computed after each generation from the best specimen and an average of all specimens. As in the simulations, we can see in Fig. 26 and Fig. 27 that after the 18th generation all values have converged to a 

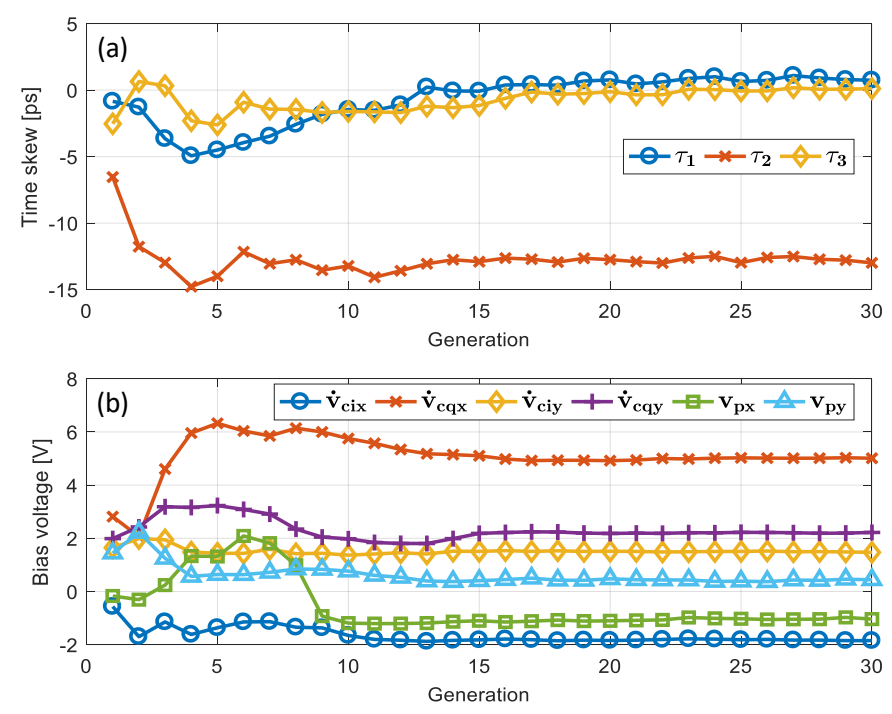

Figure 26. Experimental evolution through generations of the estimated values. (a) Time skews. (b) Children and parent bias voltages.
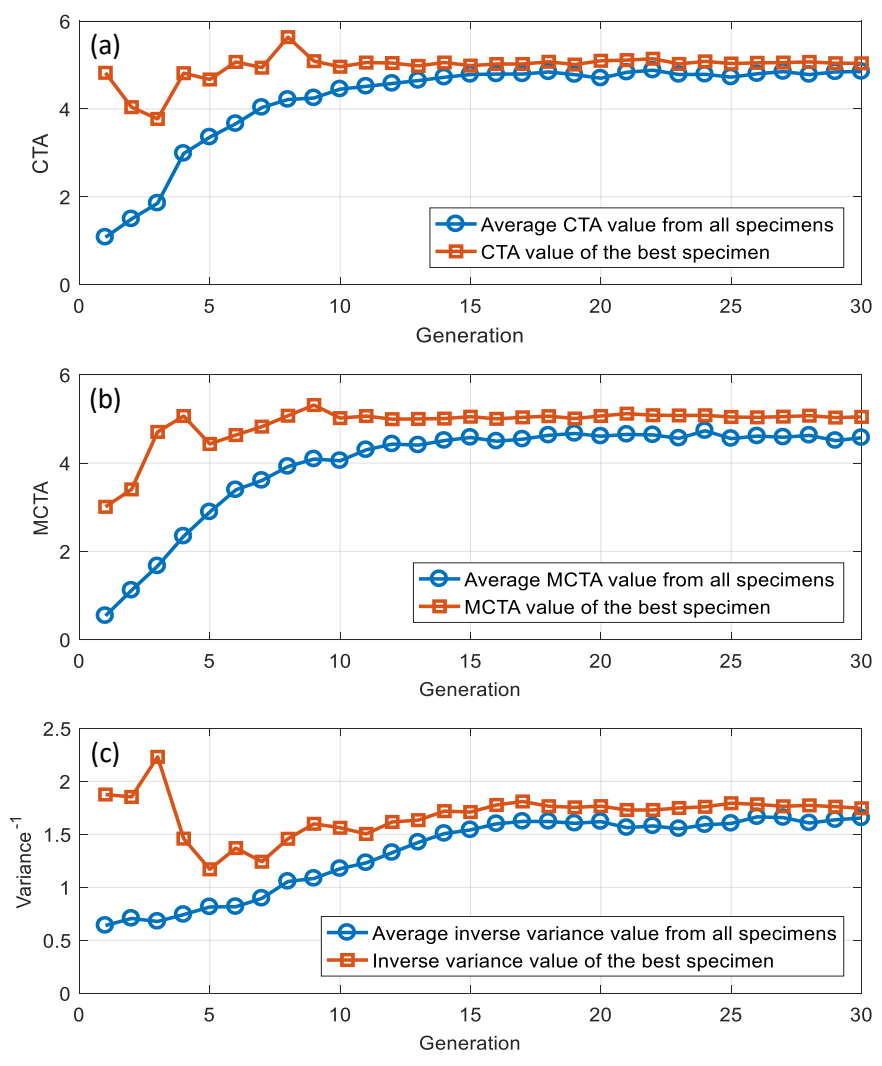

Figure 27. Experimental evolution through generations of the fitness values. (a) Clock tone amplitude. (b) Modified clock tone amplitude. (c) Inverse of the variance.

final value. Using these final values for the time skew precompensation and the bias voltages, we generated again a 16 GBd NRZ DP-16QAM and received it in a coherent receiver. After DSP offline processing, consisting of resampling to 2 samples per symbol, adaptive equalization using common $2 \times 2$ MIMO decision-directed least mean squares algorithm, and carrier recovery using blind phase search, we obtained the constellations depicted in Fig. 28.
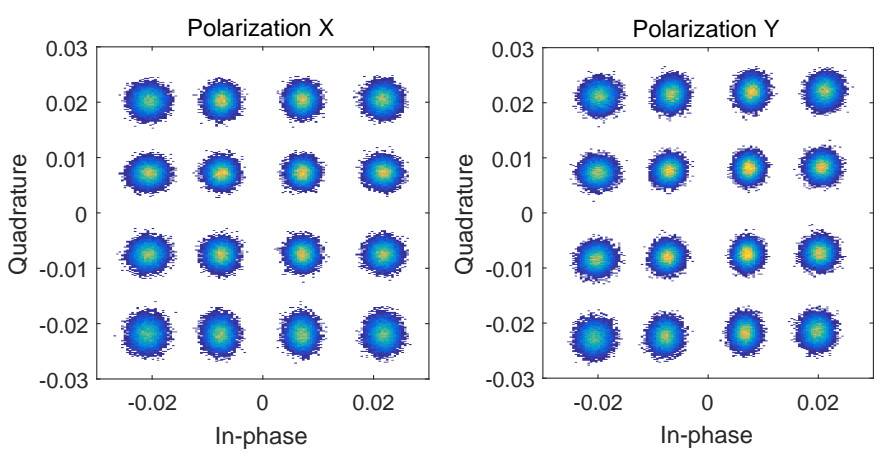

Figure 28. Obtained 16 GBd DP-16-QAM constellations after optimization using the proposed method.

Qualitatively, we can see in Fig. 28 by the recovered constellation that the bias voltages were correctly estimated, while the IQ phase had a small error of approximately $1^{\circ}$ in the polarization $\mathrm{Y}$, that was still inside the accuracy found in our simulations. This IQ phase error yields a negligible OSNR penalty for a 16QAM signal at $10^{-2}$ BER threshold and would still be less than $1 \mathrm{~dB}$ OSNR penalty if the modulation format used is changed to 256QAM [4].

\section{CONCLUSIONS}

We have presented a novel and simple method for optimization of common transmitter front-end impairments such as time skews, amplitude, and phase imbalances between inphase and quadrature components and not optimal operation point biasing. This was achieved by using a cooperative coevolutionary genetic algorithm. This method is performed in the transmitter-side, being able to optimize the transmitter for the best operation independently of the coherent receiver in an automatic way, thus avoiding the complexity increase in the already stressed receiver DSP. The performance of the presented method was numerically evaluated by simulations, and experiments were performed to demonstrate the behaviour of the method. The results also show the potential of the cooperative coevolutionary genetic algorithm as a fast optimization method to fine tune and mitigate the transmitter impairments.

\section{ACKNOWLEDGMENT}

Research leading to these results has received funding from the Villum Foundation Young Investigator program. The authors would like to thank A. C. Bravalheri for valuable discussions. The authors alone are responsible for the content.

\section{REFERENCES}

[1] E. Agrell, M. Karlsson, A. R. Chraplyvy, D. J. Richardson, P. M. Krummrich, P. Winzer, K. Roberts, J. K. Fischer, S. J. Savory, B. J. Eggleton, M. Secondini, F. R. Kschischang, A. Lord, J. Prat, I. Tomkos, J. E. Bowers, S. Srinivasan, M. Brandt-Pearce and N. Gisin, "Roadmap of optical communications," J. Opt., vol. 18, no. 6, May 2016, Art. no. 063002, 10.1088/2040-8978/18/6/063002.

[2] C. Rasmussen, Y. Pan, M. Aydinlik, M. Crowley, J. C. Geyer, P. Humblet, F. Liu, B. Mikkelsen, P. Monsen, N. Nadarajah, G. Pendock and B. Shah, "Real-time DSP for 100+ Gb/s," in Opt. Fiber Commun. Conf., Anaheim, CA, USA, 2013, paper OW1E.1, 10.1364/OFC.2013.OW1E.1.

[3] S.J. Savory, "Digital coherent optical receivers: algorithms and subsystems," IEEE J. Sel. Topics Quantum Electron., vol. 16, no. 5, pp. 1164-1179, Sept.-Oct. 2010, 10.1109/JSTQE.2010.2044751. 
[4] M. S. Faruk and S. J. Savory, "Digital Signal Processing for Coherent Transceivers Employing Multilevel Formats," J. Lightw. Technol., vol. 35, no. 5, pp. 1125-1141, Mar. 2017, 10.1109/JLT.2017.2662319.

[5] E. P. Silva and D. Zibar, "Widely Linear Equalization for IQ Imbalance and Skew Compensation in Optical Coherent Receivers," J. Lightw. Technol., vol. 34, no. 15, pp. 3577-3586, Aug. 2016, 10.1109/JLT.2016.2577716.

[6] M. Paskov, D. Lavery and S. J. Savory, "Blind Equalization of Receiver In-phase/Quadrature Skew in the Presence of Nyquist Filtering", IEEE Photon. Technol. Lett., vol. 25, no. 24, pp. 2446-2449, Dec. 2013, 10.1109/LPT.2013.2288114.

[7] R. Rios-Müller, J. Renaudier and G. Charlet, "Blind Receiver Skew Compensation and Estimation for Long-Haul Non-Dispersion Managed Systems Using Adaptive Equalizer," J. Lightw. Technol., vol. 33, no. 7, pp. 1315-1318, Apr. 2015, 10.1109/JLT.2014.2377582.

[8] C. R. S. Fludger and T. Kupfer, "Transmitter Impairment Mitigation and Monitoring for High Baud-Rate, High-order Modulation Systems", in Proc. of Eur. Conf. Opt. Commun., Düsseldorf, Germany, 2016, pp. 256-258.

[9] T.-H. Nguyen, P. Scalart, M. Gay, L. Bramerie, O. Sentieys, J.-C. Simon, C. Peucheret and M. Joindot, "Blind Transmitter IQ Imbalance Compensation in M-QAM Optical Coherent Systems," J. Opt. Commun. Netw., vol. 9, no. 9, pp. D42- D50, Sep. 2017, 10.1364/JOCN.9.000D42.

[10] N. Stojanović, and X. Chuan, "Clock Recovery in Coherent Optical Receivers", in Opt. Fiber Commun. Conf., Los Angeles, CA, 2015, paper Th3G.4, 10.1364/OFC.2015.Th3G.4.

[11] Y. Yue, B. Zhang, Q. Wang, R. Lofland, J. O'Neil, and J. Anderson, "Detection and Alignment of Dual-Polarization Optical Quadrature Amplitude Transmitter IQ and XY Skews Using Reconfigurable Interference," Opt. Express, vol. 24, no. 6, pp. 6719-6734, Mar. 2016, 10.1364/OE.24.006719.

[12] C. R. S. Fludger, T. Duthel, P. Hermann and T. Kupfer, "Low Cost Transmitter Self-Calibration of Time Delay and Frequency Response for High Baud-Rate QAM Transceivers," in Opt. Fiber Commun. Conf., Los Angeles, CA, 2017, paper Th1D.3.

[13] J. C. M. Diniz, F. Da Ros, R. T. Jones and D. Zibar, "Time Skew Estimator for Dual-Polarization QAM Transmitters," in Eur. Conf. Opt. Comm., Gothenburg, Sweden, 2017, paper P1.SC3.41.

[14] M. Seimetz, "Transmitter Design," in High-Order Modulation for Optical Fiber Transmission, Heidelberg, Germany: Springer, 2017, ch. 2, 10.1007/978-3-540-93771-5.

[15] M. Yan, Z. Tao, L. Dou, L. Li, Y. Zhao, T. Hoshida and J. C. Rasmussen, "Digital Clock Recovery Algorithm for Nyquist Signal," in Opt. Fiber Comm. Conf. Expo./Nat. Fiber Opt. Eng. Conf., Anaheim, CA, USA, paper OTu2I.7.

[16] N. Stojanović, C. Xie, Y. Zhao, B. Mao and N. Guerrero Gonzalez, "A Circuit Enabling Clock Extraction in Coherent Receivers," in Eur. Conf. Exh. Opt. Comm., Amsterdam, Netherlands, 2012, paper P3.08, 10.1364/ECEOC.2012.P3.08.

[17] L. H. H. Carvalho, C. Franciscangelis, U. R. Duarte, V. N. Rozental, J. D. Reis, F. B. Fideles, G. J. Suzigan, F. D. Simões, V. E. S. Parahyba, N. Guerrero Gonzalez, A. C. Bordonalli and J. C. R. F. Oliveira, "Multidimensional Optimization of Optical Spectral Shaping for Fiber Nonlinearities Mitigation in High Baud-Rate Systems," in Eur. Conf. Opt. Comm., Cannes, France, 2014, paper P.5.5, 10.1109/ECOC.2014.6963915.

[18] C. A. S. Diniz, M. Garrich, G. J. Suzigan, J. S. Assine, J. D. Reis, J. R. F. Oliveira and D. A. A. Mello, "Embedded System for Optical Spectral Optimization Based on a Genetic Algorithm," in SBMO/IEEE MTT-S Int. Microw. Optoelec. Conf. , Porto de Galinhas, Brazil, 2015, pp. 1-4, 10.1109/IMOC.2015.7369050.

[19] D. E. Goldberg, Genetic Algorithms in Search, Optimization and Machine Learning, Reading, MA, USA: Addison-Wesley, 1989.

[20] O. Kramer, "Genetic Algorithms," in Genetic Algorithm Essentials, Heidelberg, Germany: Springer, 2017, ch. 2, 10.1007/978-3-319-52156-5.

[21] M. A. Potter and K. A. De Jong, "Cooperative Coevolution: An Architecture for Evolving Coadapted Subcomponents," Evolutionary Computation, vol. 8, no. 1, pp. 1-29, Spring 2000, 10.1162/106365600568086. 